\title{
POSITIVE PERTURBATIONS OF SELF-ADJOINT SCHRÖDINGER OPERATORS ON RIEMANNIAN MANIFOLDS
}

\author{
OGNJEN MILATOVIC
}

\begin{abstract}
We consider a Schrödinger differential expression $L_{0}=\Delta_{M}+V_{0}$ on a (not necessarily complete) Riemannian manifold $(M, g)$ with metric $g$, where $\Delta_{M}$ is the scalar Laplacian on $M$ and $V_{0}$ is a real-valued locally square integrable function on $M$. We consider a perturbation $L_{0}+V$, where $V$ is a non-negative locally square-integrable function on $M$, and give sufficient conditions for $L_{0}+V$ to be essentially self-adjoint on $C_{c}^{\infty}(M)$. This is an extension of a result of T. Kappeler. The proof adopts Kappeler's technique, but requires the use of positivity preserving property of resolvents of certain self-adjoint operators in $L^{2}(M)$.
\end{abstract}

\section{INTRODUCTION AND THE MAIN RESULT}

1.1. The setting. Let $(M, g)$ be a $C^{\infty}$-Riemannian manifold without boundary, with metric $g=\left(g_{j k}\right)$ and $\operatorname{dim} M=n$. We will assume that $M$ is connected and oriented. We do not assume that $M$ is complete. By $d \nu$ we will denote the Riemannian volume element of $M$. In any local coordinates $x^{1}, \ldots, x^{n}$, we have $d \nu=\sqrt{\operatorname{det}\left(g_{j k}\right)} d x^{1} d x^{2} \ldots d x^{n}$.

By $L^{2}(M)$ we denote the space of complex-valued square integrable functions on $M$ with the inner product

$$
(u, v)=\int_{M}(u \bar{v}) d \nu
$$

In what follows, by $C^{\infty}(M)$ we denote the space of smooth functions on $M$, by $C_{c}^{\infty}(M)$ - the space of smooth compactly supported functions on $M$, by $\left(C_{c}^{\infty}(M)\right)^{+}$-non-negative elements of $C_{c}^{\infty}(M)$, by $\Omega^{1}(M)$ - the space of smooth 1 -forms on $M$, and by $\mathbb{Z}_{+}$- the set of positive integers.

By $d: C^{\infty}(M) \rightarrow \Omega^{1}(M)$ we denote the standard differential, and by $d^{*}: \Omega^{1}(M) \rightarrow C^{\infty}(M)$ we denote the formal adjoint of $d$ with respect to the inner product (1.1).

By $\Delta_{M}:=d^{*} d$ we will denote the scalar Laplacian on $M$.

We consider a Schrödinger-type differential expression

$$
L_{0}=\Delta_{M}+V_{0},
$$

where $V_{0} \in L_{\text {loc }}^{2}(M)$ is a real-valued function.

1.2. Operators $H_{0}$ and $T_{0}$. Define an operator $H_{0}$ by the formula $H_{0} u=L_{0} u$ with the domain $\operatorname{Dom}\left(H_{0}\right)=C_{c}^{\infty}(M)$. Since $H_{0}$ is a symmetric operator in $L^{2}(M)$, it follows that $H_{0}$ is closable. Let $T_{0}$ be the closure $\widetilde{H}_{0}$ of $H_{0}$.

2000 Mathematics Subject Classification. Primary 35P05, 58J50; Secondary 47B25, 81Q10. 
Assumption A. Assume that $V \in L_{\mathrm{loc}}^{2}(M)$ and $V \geq 0$.

1.3. Operators $H$ and $T$. Let $V$ be as in Assumption (A). Define an operator $H$ by the formula $H u=L_{0} u+V u$ with the domain $\operatorname{Dom}(H)=C_{c}^{\infty}(M)$. Since $H$ is a symmetric operator in $L^{2}(M)$, it follows that $H$ is closable. Let $T$ be the closure $\widetilde{H}$ of $H$.

We make an additional Assumption on $V$.

Assumption B. Assume that there exist constants $a \geq 0$ and $b \geq 0$ such that

$$
\|V u\| \leq a\left\|T_{0} u\right\|+b\|u\|, \quad \text { for all } u \in C_{c}^{\infty}(M),
$$

where $\|\cdot\|$ denotes the norm in $L^{2}(M)$ corresponding to (1.1).

We now state the main results.

Theorem 1.4. Assume that $(M, g)$ is a (not necessarily complete) $C^{\infty}$-Riemannian manifold without boundary. Assume that $M$ is connected and oriented. Assume that the operator $T_{0}$ is semi-bounded below and self-adjoint. Assume that $V$ satisfies Assumptions (A) and (B). Then the operator $T$ is self-adjoint.

Corollary 1.5. Assume that the hypotheses of Theorem 1.4 are satisfied. Additionally, assume that $T_{0}$ satisfies the following property:

(P) for every $0 \leq u \in \operatorname{Dom}\left(T_{0}\right)$, there exists a sequence $\left\{u_{k}\right\}$ in $\left(C_{c}^{\infty}(M)\right)^{+}$such that $u_{k} \rightarrow u$ and $T_{0} u_{k} \rightarrow T_{0} u$ in $L^{2}(M)$, as $k \rightarrow \infty$.

Then $T$ also satisfies the property $(P)$ (with $T_{0}$ replaced by $T$ ).

Remark 1.6. Theorem 1.4 and Corollary 1.5 extend the results of T. Kappeler (see Theorem 1 and Corollary 3 in [4]) concerning the Schrödinger differential expressions $L_{0}=-\Delta+V_{0}$ and $L_{0}+V$, where $\Delta$ is the standard Laplacian on an open set $\Omega \subset \mathbb{R}^{n}$ and $V_{0}$ and $V$ are as in the hypotheses of Theorem 1.4.

\section{Proof of Theorem 1.4}

Throughout this section, we assume that all hypotheses of Theorem 1.4 are satisfied. By using positivity preserving property of resolvents of certain self-adjoint operators in $L^{2}(M)$, we will adopt Kappeler's technique from [4] to our setting.

We know by hypothesis that $T_{0}$ is semi-bounded below. Without loss of generality we may and we will assume that $T_{0}$ is non-negative, i.e.

$$
\left(T_{0} u, u\right) \geq 0, \quad \text { for all } u \in \operatorname{Dom}\left(T_{0}\right) .
$$

Since $0 \leq V \in L_{\text {loc }}^{2}(M)$, by definition of $T$ it follows that $T$ is a symmetric and non-negative operator. Let $T_{F}$ denote the Friedrichs extension of $T$; see, for example, Section VI.2.3 in Kato [5]. Since $T_{F}$ is a self-adjoint extension of $T$, to prove the Theorem, it suffices to show that $\operatorname{Dom}\left(T_{F}\right) \subset \operatorname{Dom}(T)$.

The following Lemma provides a key step in the proof of the Theorem. 
Lemma 2.1. Assume that $u \in \operatorname{Dom}\left(T_{F}\right)$. Then there exists a sequence $\left\{u_{k}\right\}$ in $\operatorname{Dom}\left(T_{0}\right)$ such that

$$
u_{k} \rightarrow u \quad \text { and } \quad T_{F} u_{k} \rightarrow T_{F} u \quad \text { in } L^{2}(M), \quad \text { as } k \rightarrow \infty .
$$

Remark 2.2. In Lemma 2.14 below, we will show that $\operatorname{Dom}\left(T_{0}\right) \subset \operatorname{Dom}(T)$, so that $T_{F} u_{k}$ in $(2.1)$ is a sequence in $L^{2}(M)$ (because $\operatorname{Dom}(T) \subset \operatorname{Dom}\left(T_{F}\right)$ ).

In the proof of Lemma 2.1, we will use the following notations and Lemmas.

2.3. Potentials $V_{m}$. For every $m \in \mathbb{Z}_{+}$and $x \in M$, define

$$
V_{m}(x)= \begin{cases}V(x) & \text { if } V(x) \leq m, \\ m & \text { if } V(x)>m .\end{cases}
$$

2.4. Operator $T_{m}$. Since $V_{m} \in L^{\infty}(M)$, the multiplication operator $\left(V_{m} u\right)(x)=V_{m}(x) u(x)$ in $L^{2}(M)$ is defined for all $u \in L^{2}(M)$. We define an operator $T_{m}$ in $L^{2}(M)$ by the formula $T_{m}=T_{0}+V_{m}$ with the domain $\operatorname{Dom}\left(T_{m}\right)=\operatorname{Dom}\left(T_{0}\right) \cap \operatorname{Dom}\left(V_{m}\right)=\operatorname{Dom}\left(T_{0}\right)$.

Lemma 2.5. For all $m \in \mathbb{Z}_{+}$, the operator $T_{m}$ is self-adjoint. Moreover, $T_{m}$ is the closure of $\left.\left(\Delta_{M}+V_{0}+V_{m}\right)\right|_{C_{c}^{\infty}(M)}$, and

$$
\left(T_{m} u, u\right) \geq\left(T_{0} u, u\right) \geq 0, \quad \text { for all } u \in \operatorname{Dom}\left(T_{m}\right)=\operatorname{Dom}\left(T_{0}\right) .
$$

Proof. Since $V_{m} \in L^{\infty}(M)$, the multiplication operator $V_{m}$ is $T_{0}$-bounded with the relative bound 0. Thus, by Theorem V.4.4 in [5], it follows that $T_{m}$ is self-adjoint.

Since the multiplication operator $V_{m}$ is $H_{0}$-bounded with relative bound 0 and since $H_{0}$ (with $\left.\operatorname{Dom}\left(H_{0}\right)=C_{c}^{\infty}(M)\right)$ is essentially self-adjoint (because $T_{0}=\widetilde{H_{0}}$ is self-adjoint by hypothesis), by Theorem V.4.4 in [5] it follows that $H_{0}+V_{m}$ is essentially self-adjoint on $C_{c}^{\infty}(M)$ and $\left(H_{0}+V_{m}\right)^{\sim}=\widetilde{H_{0}}+\widetilde{V_{m}}$, where $V_{m}$ is the multiplication operator as in Subsection 2.4. Since $\operatorname{Dom}\left(V_{m}\right)=L^{2}(M)$ and since $V_{m}$ is a bounded linear operator in $L^{2}(M)$, it follows that the operator $V_{m}$ is closed. Thus, we have

$$
T_{m}=T_{0}+V_{m}=\widetilde{H_{0}}+\widetilde{V_{m}}=\left(H_{0}+V_{m}\right)^{\sim}=\left(\left.\left(\Delta_{M}+V_{0}+V_{m}\right)\right|_{C_{c}^{\infty}(M)}\right)^{\sim} .
$$

Since $V_{m} \geq 0$ and since $T_{0}$ is a non-negative operator, the inequality (2.2) immediately follows, and the Lemma is proven.

Since $T_{m}$ and $T_{0}$ are non-negative self-adjoint operators, it follows that for all $k, m \in \mathbb{Z}_{+}$, the operators

$$
\left(\frac{T_{m}}{k}+1\right)^{-1}: L^{2}(M) \rightarrow L^{2}(M) \quad \text { and } \quad\left(\frac{T_{0}}{k}+1\right)^{-1}: L^{2}(M) \rightarrow L^{2}(M)
$$

are bounded linear operators; see, for example, Section V.3.10 in [5]. 
2.6. Positivity Preserving Property. For the following definition, see, for example, the Definition below the formulation of Theorem X.30 in [7].

Definition 2.7. Let $(X, \mu)$ be a measure space. A bounded linear operator $A: L^{2}(X, \mu) \rightarrow$ $L^{2}(X, \mu)$ is said to be positivity preserving if for every $u \in L^{2}(X, \mu)$ such that $u \geq 0$ a.e. on $X$, we have $A u \geq 0$ a.e. on $X$.

Remark 2.8. Let $A: L^{2}(X, \mu) \rightarrow L^{2}(X, \mu)$ be a positivity preserving bounded linear operator. Then the following inequality holds for all $u \in L^{2}(X, \mu)$ :

$$
|(A u)(x)| \leq A|u(x)|, \quad \text { a.e. on } X,
$$

where $|\cdot|$ denotes the absolute value of a complex number. For the proof of (2.4), see the proof of the inequality (X.103) in [7].

An important ingredient in the proof of Lemma 2.1 is the following Proposition.

Proposition 2.9. Assume that $(M, g)$ is a (not necessarily complete) $C^{\infty}$-Riemannian manifold without boundary. Assume that $M$ is connected and oriented. Assume that $Q_{0} \in L_{\mathrm{loc}}^{2}(M)$ is real-valued. Additionally, assume that

$$
\left(\left(\Delta_{M}+Q_{0}\right) u, u\right) \geq 0, \quad \text { for all } u \in C_{c}^{\infty}(M) .
$$

Let $S_{0}$ be the Friedrichs extension of $\left.\left(\Delta_{M}+Q_{0}\right)\right|_{C_{c}^{\infty}(M)}$. Assume that $\lambda$ is a positive real number. Then the operator $\left(S_{0}+\lambda\right)^{-1}$ is positivity preserving.

Remark 2.10. For the proof of Proposition 2.9, which is based on Kato's inequality technique on Riemannian manifolds, see the proof of Proposition 2.13 in [6]. Proposition 2.9 is an extension to Riemannian manifolds of Lemma 2 from Goelden [3]. For more on Kato's inequality technique on Riemannian manifolds and its application to essential self-adjointness of Schrödinger-type operators, see [1] and references there.

Corollary 2.11. The operators in (2.3) are positivity preserving.

Proof. Let $k, m \in \mathbb{Z}_{+}$. It suffices to show that the operators $\left(T_{0}+k\right)^{-1}$ and $\left(T_{m}+k\right)^{-1}$ are positivity preserving. By hypothesis and the definition of $T_{0}$, the operator $T_{0}$ is the self-adjoint closure of $H_{0}$. Thus, $T_{0}$ is the Friedrichs extension of $H_{0}$; hence, by Proposition 2.9, the operator $\left(T_{0}+k\right)^{-1}$ is positivity preserving. Since $0 \leq V_{m} \in L^{\infty}(M)$ and since $T_{0}$ is non-negative, it follows that $Q_{m}:=V_{0}+V_{m}$ satisfies the hypotheses of Proposition 2.9. By Lemma 2.5 it follows that $T_{m}$ is the self-adjoint closure of $\left.\left(\Delta_{M}+Q_{m}\right)\right|_{C_{c}^{\infty}(M)}$. Thus, $T_{m}$ is the Friedrichs extension of $\left.\left(\Delta_{M}+Q_{m}\right)\right|_{C_{c}^{\infty}(M)}$; hence, by Proposition 2.9 , the operator $\left(T_{m}+k\right)^{-1}$ is positivity preserving. This concludes the proof.

2.12. Sequence $v_{k m}$. Let $u \in \operatorname{Dom}\left(T_{F}\right)$ and let $k, m \in \mathbb{Z}_{+}$. Define the following sequence:

$$
v_{k m}:=\left(\frac{T_{m}}{k}+1\right)^{-1} u \text {. }
$$

Since $u \in \operatorname{Dom}\left(T_{F}\right) \subset L^{2}(M)$, by (2.3) it follows that the sequence $v_{k m}$ is well-defined. Moreover, we have $v_{k m} \in \operatorname{Dom}\left(T_{m}\right)=\operatorname{Dom}\left(T_{0}\right)$. 
Lemma 2.13. Let $u$ and $v_{k m}$ be as in (2.5). Then

$$
\left|v_{k m}\right| \leq\left(\frac{T_{0}}{k}+1\right)^{-1}|u|
$$

Proof. By Corollary 2.11, the operator $\left(\left(T_{m} / k\right)+1\right)^{-1}$ is positivity preserving. Hence, by $(2.4)$ we get

$$
\left|v_{k m}\right| \leq\left(\frac{T_{m}}{k}+1\right)^{-1}|u|
$$

where $v_{k m}$ and $u$ are as in (2.5).

To prove (2.6), we will show that

$$
\left(\frac{T_{m}}{k}+1\right)^{-1}|u| \leq\left(\frac{T_{0}}{k}+1\right)^{-1}|u|
$$

We have

$$
\begin{aligned}
\left(\frac{T_{0}}{k}+1\right)^{-1}|u| & -\left(\frac{T_{m}}{k}+1\right)^{-1}|u|= \\
=\left(\frac{T_{0}}{k}+1\right)^{-1}\left(\left(\frac{T_{m}}{k}+1\right)\right. & \left.-\left(\frac{T_{0}}{k}+1\right)\right)\left(\frac{T_{m}}{k}+1\right)^{-1}|u| \\
& =\left(\frac{T_{0}}{k}+1\right)^{-1}\left(\frac{V_{m}}{k}\right)\left(\frac{T_{m}}{k}+1\right)^{-1}|u| .
\end{aligned}
$$

Since $0 \leq V_{m} \in L^{\infty}(M)$, by Corollary 2.11 it follows that the right hand side of the last inequality in (2.8) is non-negative. This shows (2.7), and the Lemma is proven.

Lemma 2.14. Under the hypotheses of Theorem 1.4, the following hold:

(i) $\operatorname{Dom}\left(T_{0}\right) \subset \operatorname{Dom}(T)$.

(ii) For all $b \in \operatorname{Dom}\left(T_{0}\right)$, we have

$$
T_{F} b=T_{0} b+V b,
$$

where $V$ is understood as the maximal multiplication operator with

$$
\operatorname{Dom}(V)=\left\{u \in L^{2}(M): V u \in L^{2}(M)\right\} .
$$

Proof. We first prove part (i). Let $b \in \operatorname{Dom}\left(T_{0}\right)$. Since $T_{0}=\widetilde{H_{0}}$ and since $\operatorname{Dom}\left(H_{0}\right)=C_{c}^{\infty}(M)$, it follows that there exists a sequence $b_{k} \in C_{c}^{\infty}(M)$ such that $b_{k} \rightarrow b$ in $L^{2}(M)$ and $H_{0} b_{k} \rightarrow T_{0} b$ in $L^{2}(M)$, as $k \rightarrow \infty$; see, for example, Section III.5.3 in [5]. Hence, by (1.2) it follows that the sequence $\left\{V b_{k}\right\}$ is a Cauchy sequence in $L^{2}(M)$. Therefore, the sequence $\left\{H_{0} b_{k}+V b_{k}\right\}$ is a Cauchy sequence in $L^{2}(M)$. By the definition of $T$ in Subsection 1.3, we have $T=\widetilde{H}$. Thus, since $b_{k} \rightarrow b$ in $L^{2}(M)$ and since $\left\{H b_{k}\right\}=\left\{H_{0} b_{k}+V b_{k}\right\}$ is a Cauchy sequence in $L^{2}(M)$, it follows that $b \in \operatorname{Dom}(T)$ (and $T b=\lim _{k \rightarrow \infty} H b_{k}$ in $L^{2}(M)$ ). This concludes the proof of part (i).

We now prove part (ii). Let $b \in \operatorname{Dom}\left(T_{0}\right)$ and let $\left\{b_{k}\right\}$ be the sequence as in the proof of part (i) of this Lemma. It is well-known the maximal multiplication operator $V$ is self-adjoint (hence, closed); see, for example, Problem V.3.22 in [5]. By the proof of part (i), we know that 
$b_{k} \in C_{c}^{\infty}(M) \subset \operatorname{Dom}(V)$ and $b_{k} \rightarrow b$ in $L^{2}(M)$ and $\left\{V b_{k}\right\}$ is a Cauchy sequence in $L^{2}(M)$. Since $V$ is closed, it follows that $V b_{k} \rightarrow V b$ in $L^{2}(M)$, as $k \rightarrow \infty$. Since $b \in \operatorname{Dom}\left(T_{0}\right) \subset \operatorname{Dom}(T)$ and since $T_{F}$ is an extension of $T$, we have $T_{F} b=T b$. By the proof of part (i) we have

$$
T_{F} b=T b=\lim _{k \rightarrow \infty}\left(H b_{k}\right)=\lim _{k \rightarrow \infty}\left(H_{0} b_{k}\right)+\lim _{k \rightarrow \infty} V b_{k}=T_{0} b+V b,
$$

where the limits in (2.10) denote the convergence in $L^{2}(M)$.

This shows (2.9), and the Lemma is proven.

Lemma 2.15. Let $u$ and $v_{k m}$ be as in (2.5). Let $k \in \mathbb{Z}_{+}$be fixed. Then, the following holds:

$$
v_{k m} \rightarrow\left(\frac{T_{F}}{k}+1\right)^{-1} u \quad \text { in } L^{2}(M), \quad \text { as } m \rightarrow \infty .
$$

Proof. Let $k \in \mathbb{Z}_{+}$be fixed. Since $v_{k m} \in \operatorname{Dom}\left(T_{m}\right)=\operatorname{Dom}\left(T_{0}\right)$, by Lemma 2.14 we have

$$
T_{F} v_{k m}=T_{0} v_{k m}+V v_{k m} .
$$

Therefore,

$$
\begin{aligned}
v_{k m}-\left(\frac{T_{F}}{k}+1\right)^{-1} u=\left(\frac{T_{F}}{k}+1\right)^{-1}\left(\left(\frac{T_{F}}{k}+1\right)\right. & \left.-\left(\frac{T_{m}}{k}+1\right)\right) v_{k m}= \\
& =\left(\frac{T_{F}}{k}+1\right)^{-1}\left(\frac{V-V_{m}}{k}\right) v_{k m} .
\end{aligned}
$$

Since $T_{F}$ is non-negative and self-adjoint, it follows that $\left(\left(T_{F} / k\right)+1\right)^{-1}: L^{2}(M) \rightarrow L^{2}(M)$ is a bounded linear operator. Thus, to finish the proof of the Lemma, it is enough to show that

$$
\left(V-V_{m}\right) v_{k m} \rightarrow 0 \quad \text { in } L^{2}(M), \quad \text { as } m \rightarrow \infty .
$$

By (2.6) and by the definition of $V_{m}$ it follows that

$$
\left(V-V_{m}\right) v_{k m} \rightarrow 0 \quad \text { a.e. on } M, \quad \text { as } m \rightarrow \infty .
$$

Moreover, by Lemma 2.13 we have

$$
\left|\left(V-V_{m}\right) v_{k m}\right|=\left(V-V_{m}\right)\left|v_{k m}\right| \leq\left(V-V_{m}\right)\left(\frac{T_{0}}{k}+1\right)^{-1}|u| \leq V\left(\frac{T_{0}}{k}+1\right)^{-1}|u| .
$$

The last inequality in (2.15) follows by the definition of $V_{m}$.

Since, by hypothesis, $H_{0}=\left.\left(\Delta_{M}+V_{0}\right)\right|_{C_{c}^{\infty}(M)}$ is an essentially self-adjoint operator with closure $T_{0}=\widetilde{H_{0}}$ and since $\left(\left(\left(T_{0} / k\right)+1\right)^{-1}|u|\right) \in \operatorname{Dom}\left(T_{0}\right)$, by using $(1.2)$ and by repeating the same arguments as in the beginning of the proof of part (ii) of Lemma 2.14, it follows that $\left(V\left(\left(T_{0} / k\right)+1\right)^{-1}|u|\right) \in L^{2}(M)$.

Using (2.14), (2.15) and Dominated Convergence Theorem, we obtain (2.13). This concludes the proof of the Lemma. 
Lemma 2.16. Let $u$ and $v_{k m}$ be as in (2.5). Let $k \in \mathbb{Z}_{+}$be fixed. Then, the following holds:

$$
T_{F} v_{k m} \rightarrow T_{F}\left(\frac{T_{F}}{k}+1\right)^{-1} u \quad \text { in } L^{2}(M), \quad \text { as } m \rightarrow \infty .
$$

Proof. Since $v_{k m} \in \operatorname{Dom}\left(T_{m}\right)=\operatorname{Dom}\left(T_{0}\right)$, by Lemma 2.14 we have

$$
T_{F} v_{k m}=T_{0} v_{k m}+V v_{k m}=T_{0} v_{k m}+V_{m} v_{k m}+\left(V-V_{m}\right) v_{k m}=T_{m} v_{k m}+\left(V-V_{m}\right) v_{k m} .
$$

By the proof of $(2.13)$, we have $\left(V-V_{m}\right) v_{k m} \rightarrow 0$ in $L^{2}(M)$ as $m \rightarrow \infty$.

Writing

$$
T_{m} v_{k m}=k\left(\frac{T_{m}}{k}+1\right) v_{k m}-k v_{k m}=k u-k v_{k m},
$$

and using (2.11), we have, as $m \rightarrow \infty$ :

$$
T_{m} v_{k m} \rightarrow k u-k\left(\frac{T_{F}}{k}+1\right)^{-1} u \quad \text { in } L^{2}(M)
$$

Since

$$
k u-k\left(\frac{T_{F}}{k}+1\right)^{-1} u=T_{F}\left(\frac{T_{F}}{k}+1\right)^{-1} u
$$

the Lemma is proven.

Lemma 2.17. Let $u$ be as in (2.5). Then, as $k \rightarrow \infty$, we have

$$
\left(\frac{T_{F}}{k}+1\right)^{-1} u \rightarrow u \quad \text { and } \quad T_{F}\left(\frac{T_{F}}{k}+1\right)^{-1} u \rightarrow T_{F} u \quad \text { in } L^{2}(M) .
$$

Proof. Let $k \in \mathbb{Z}_{+}$. By hypothesis, the (non-negative self-adjoint) operator $T_{F} / k$ is the Friedrichs extension of $T / k$. Let $t_{F, k}$ be the (densely defined, closed and non-negative) quadratic form associated to $T_{F} / k$ by Theorem VI.2.7 in [5]. Since $t_{F, k}$ is non-negative, we have the following inequality of quadratic forms:

$$
t_{F, k} \geq 0
$$

where 0 on the right hand side denotes the zero-form $0(\cdot, \cdot)$ with the domain $L^{2}(M)$.

Since $C_{c}^{\infty}(M) \subset \operatorname{Dom}(T / k) \subset \operatorname{Dom}\left(T_{F} / k\right)$, we have

$$
\left(\frac{T_{F}}{k} s, s\right)=\frac{1}{k}\left(T_{F} s, s\right) \rightarrow 0=(0 s, s), \quad \text { for all } s \in C_{c}^{\infty}(M), \quad \text { as } k \rightarrow \infty,
$$

where 0 in $(0 s, s)$ is the zero operator $0: L^{2}(M) \rightarrow L^{2}(M)$.

Since by Theorem VI.2.1 in [5], the domain $\operatorname{Dom}\left(T_{F} / k\right) \subset \operatorname{Dom}\left(t_{F, k}\right)$, it follows that $C_{c}^{\infty}(M) \subset$ $\operatorname{Dom}\left(t_{F, k}\right)$. We also know that $C_{c}^{\infty}(M)$ is dense in $\operatorname{Dom}(0(\cdot, \cdot))=L^{2}(M)$. This, together with (2.18) and (2.19), shows that the hypotheses of abstract Theorem 7.9 from [2] are satisfied.

By Theorem 7.9 from [2] it follows that $T_{F} / k \rightarrow 0$ in the strong resolvent sense, as $k \rightarrow \infty$. Since -1 belongs to the resolvent sets of operators $T_{F} / k$ and 0 , we have

$$
\left(\frac{T_{F}}{k}+1\right)^{-1} \rightarrow 1, \quad \text { as } k \rightarrow \infty
$$


strongly (as a sequence of bounded linear operators $L^{2}(M) \rightarrow L^{2}(M)$ ). Here, $1: L^{2}(M) \rightarrow$ $L^{2}(M)$ denotes the identity operator defined on the whole $L^{2}(M)$.

Thus, by the definition of strong convergence (for sequences of bounded linear operators), the leftmost convergence relation in (2.17) follows from (2.20).

We know that $T_{F}$ is a non-negative self-adjoint operator. Since -1 belongs to the resolvent set of $T_{F} / k$ and since $u \in \operatorname{Dom}\left(T_{F}\right)$, by Problem III.6.2 in [5] it follows that

$$
T_{F}\left(\frac{T_{F}}{k}+1\right)^{-1} u=\left(\frac{T_{F}}{k}+1\right)^{-1} T_{F} u \text {. }
$$

Since $T_{F} u \in L^{2}(M)$, the rightmost convergence relation in (2.17) follows from (2.21) and (2.20). This concludes the proof of the Lemma.

Proof of Lemma 2.1. By (2.11) and (2.16), for every $k \in \mathbb{Z}_{+}$, we can choose $m(k)$ such that

$$
\left\|v_{k, m(k)}-\left(\frac{T_{F}}{k}+1\right)^{-1} u\right\| \leq \frac{1}{k} .
$$

and

$$
\left\|T_{F} v_{k, m(k)}-T_{F}\left(\frac{T_{F}}{k}+1\right)^{-1} u\right\| \leq \frac{1}{k} .
$$

Let $u_{k}:=v_{k, m(k)}$. Then the sequence $\left\{u_{k}\right\}$ is in $\operatorname{Dom}\left(T_{0}\right)$, and (2.22) and (2.23) hold with $v_{k, m(k)}$ and replaced by $u_{k}$.

Using (2.22) and (2.23) (with $v_{k, m(k)}$ and replaced by $u_{k}$ ), and using (2.17), it follows that $\left\{u_{k}\right\}$ satisfies the properties (2.1). This concludes the proof of the Lemma.

Proof of Theorem 1.4. Let $u \in \operatorname{Dom}\left(T_{F}\right)$. By Lemma 2.1 there exists a sequence $\left\{u_{k}\right\}$ in $\operatorname{Dom}\left(T_{0}\right)$ satisfying (2.1). By Lemma 2.14 it follows that $\operatorname{Dom}\left(T_{0}\right) \subset \operatorname{Dom}(T)$. Since $T \subset T_{F}$, from (2.1) it follows that $\left\{u_{k}\right\}$ is a sequence in $\operatorname{Dom}\left(T_{0}\right) \subset \operatorname{Dom}(T)$ such that $u_{k} \rightarrow u$ in $L^{2}(M)$ and $\left\{T u_{k}\right\}$ converges in $L^{2}(M)$. Since, by hypothesis, $T$ is a closed operator, it follows that $u \in \operatorname{Dom}(T)$. This shows that $\operatorname{Dom}\left(T_{F}\right) \subset \operatorname{Dom}(T)$, and the Theorem is proven.

\section{Proof of Corollary 1.5}

Throughout this section, we assume that all hypotheses of Corollary 1.5 are satisfied.

We begin with the following Lemma.

Lemma 3.1. Assume that $0 \leq u \in \operatorname{Dom}(T)$. Then, there exists a sequence $\left\{u_{k}\right\} \in \operatorname{Dom}\left(T_{0}\right)$ such that $u_{k} \geq 0$ and

$$
u_{k} \rightarrow u, \quad \text { and } \quad T u_{k} \rightarrow T u \quad \text { in } L^{2}(M), \quad \text { as } k \rightarrow \infty .
$$

Proof. By Theorem 1.4 the operator $T$ is self-adjoint (and $T=T_{F}$ ). We define $v_{k m} \in \operatorname{Dom}\left(T_{0}\right)$ as in (2.5). Since $u \geq 0$, by Corollary 2.11 it follows that $v_{k m} \geq 0$. Let $u_{k}:=v_{k, m(k)}$ be as in the proof of Lemma 2.1. By the proof of Lemma 2.1, it follows that the sequence $\left\{u_{k}\right\}$ satisfies the properties (3.1). 
Proof of Corollary 1.5. Let $0 \leq u \in \operatorname{Dom}(T)$. By Lemma 3.1, for any $\epsilon>0$, there exists a function $0 \leq w \in \operatorname{Dom}\left(T_{0}\right)$ such that

$$
\|w-u\|<\epsilon \quad \text { and } \quad\|T w-T u\|<\epsilon .
$$

By hypotheses of the Corollary, the operator $T_{0}$ satisfies the property $(\mathrm{P})$. Thus, for any $\epsilon>0$, there exists a function $s \in\left(C_{c}^{\infty}(M)\right)^{+}$such that

$$
\|s-w\|<\epsilon \quad \text { and } \quad\left\|T_{0} s-T_{0} w\right\|<\epsilon .
$$

By hypotheses of the Corollary, the function $V$ satisfies (1.2). By the proof of the inequality (4.6) from Section V.4.1 from [5], it follows that (1.2) holds for all $u \in \operatorname{Dom}\left(T_{0}\right)$ (where $V$ is understood as in part (ii) of Lemma 2.14). Therefore,

$$
\|V(s-w)\| \leq a\left\|T_{0}(s-w)\right\|+b\|s-w\|,
$$

where $a$ and $b$ are as in (1.2).

Using, (3.2), (3.3) and (3.4), we obtain

$$
\|s-u\|<2 \epsilon
$$

and

$$
\begin{array}{r}
\|T s-T u\|=\|(T s-T w)+(T w-T u)\|=\left\|\left(\left(T_{0} s+V s\right)-\left(T_{0} w+V w\right)\right)+(T w-T u)\right\| \leq \\
\leq\left\|T_{0} s-T_{0} w\right\|+\|V s-V w\|+\|T w-T u\|<(2+a+b) \epsilon . \quad
\end{array}
$$

On the right hand side of the second equality in (3.6) we used (2.9) with $T_{F}=T$.

By (3.5) and (3.6), it follows that the operator $T$ satisfies the property $(\mathrm{P})$. This concludes the proof of the Corollary.

\section{REFERENCES}

[1] M. Braverman, O. Milatovic, M. Shubin, Essential self-adjointness of Schrödinger type operators on manifolds, Russian Math. Surveys, 57(4) (2002), 641-692.

[2] W. G. Faris, Self-adjoint Operators, Lecture Notes in Mathematics No. 433, Springer-Verlag, Berlin e.a., 1975.

[3] H.-W. Goelden, On non-degeneracy of the ground state of Schrödinger operators, Math. Z., 155 (1977), 239-247.

[4] T. Kappeler, Positive perturbations of self-adjoint Schrödinger operators, Proc. Royal Soc. Edinburgh, 99A (1985), 241-248.

[5] T. Kato, Perturbation Theory For Linear Operators, Springer-Verlag, New York, 1980.

[6] O. Milatovic, The form sum and the Friedrichs extension of Schrödinger-type operators on Riemannian manifolds, Proc. Amer. Math. Soc., 132 (1) (2004), 147-156.

[7] M. Reed, B. Simon, Methods of Modern Mathematical Physics I, II: Functional analysis. Fourier analysis, self-adjointness, Academic Press, New York e.a., 1975.

Department of Mathematics and Statistics, University of North Florida, Jacksonville, FL 32224, USA

E-mail address: omilatov@unf.edu 